\title{
Electromyography Data Transmission via Galvanic Coupling Intra-body Communication Link
}

\author{
Anna Vizziello, Senior Member, IEEE, Pietro Savazzi, Senior Member, IEEE, and Giovanni Magenes, Senior \\ Member, IEEE
}

\begin{abstract}
Intra-body communication (IBC) is a novel research filed that will promote personalized medicine by allowing real time and in situ monitoring in daily life. In this work, the energy efficient galvanic coupling (GC) technology is used to send electromyography (EMG) data through intra-body links. Real EMG data are first acquired and recorded with needle electrodes inserted in the muscle of a person's forearm. Then, the data are transferred via GC communication employing a GC sound card-based testbed. The experiments are conducted by transferring EMG data in both ex-vivo and in-vivo tissue with different electrodes placements. Almost error free performance is achieved with a robust and reliable communication, a valuable result in medical applications.
\end{abstract}

Index Terms-Intra-body networks, intra-body communication, galvanic coupling technology, coupling circuits, wireless sensor network, biomedical engineering, biomedical electronics, experimental testbed.

\section{INTRODUCTION}

I MPLANTED sensors will foster next generation healthcare by allowing real time physiological monitoring, proactive drug delivery and personalized medicine. Intra-body network (IBN) concept enable the interconnection of implants and allows them to transmit the sensed measurements to an external monitoring center for processing, in order to receive updates on the amount of drug delivery and to send commands to embedded actuators.

On this purpose, energy efficient communication are required between wearable sensors placed on the body and an external device for monitoring and processing and among implants for data communication. For the first scenario, Zigbee, ANT and Bluetooth have been profitably used in body area networks (BANs) [1]. For the second scenario of intrabody communication, several studies have been conducted to evaluate the signal strength of both narrowband radio frequency (RF) signals and ultra wide band (UWB) ones along and inside the human body, which confirmed that the radio signals show high losses [2], [3]. This results in undesired tissues heating and energy waste for the implanted sensors. Alternative non-RF solutions are thus needed.

Non-RF techniques using the human body as a medium for data communication include ultrasound (US) [4], capacitive coupling (CC), inductive coupling (IC) and galvanic coupling (GC) [1]. We employ GC technology with low/medium frequency $(1 \mathrm{KHz}-100 \mathrm{MHz})$, where weak $(<1 \mathrm{~mA})$ electrical

A. Vizziello, P. Savazzi and G. Magenes are with the Department of Electrical, Computer and Biomedical Engineering, University of Pavia, 27100, Pavia Italy (Corresponding author: Anna Vizziello, e-mail: anna.vizziello@unipv.it). currents are modulated with data and a couple of electrodes are used at both the transmitter and the receiver, as described in Sec. II.

Existing GC works analyzed the signal strength within the tissues [1], [5], [6] and modelled human tissues as communication channel [1], [7], [8]. Some GC testbeds have been also designed and implemented to establish an intra-body communication link. They include a complex programmable logic device (CPLD) with a field-programmable gate array (FPGA) at the receiver [5]; a FPGA board with an impulse radio (IR) and a pulse position modulation (PPM) [9]; universal software radio peripherals (USRPs) with low frequency daughterboards [10]; and a ultra-low-power solution with off-theshelf electronic components [11]. However, a quick repeatable GC platform to conduct experiments is still a challenge.

We here employ the GC platform that we have developed in [12], [13], whose main features are its easy repeatability and physical layer (PHY) methods able to obtain almost error free performance. The GC testbed [12], [13] leverages on PC sound card and Matlab software since GC frequency range $(1 \mathrm{KHz}$ - $100 \mathrm{MHz}$ ) [6] includes the signals' frequencies supported by the sound cards. A Matlab session must be open in each of the two PC to run the transmitter and receiver software respectively. The sound cards are used to allow real signal transmission/reception. Such signal is then sent out from the PC to an biological tissue through LINE IN/ LINE OUT jack that are connected to the electrodes to inject currents inside the tissue (Fig. 5). PHY methods are implemented in the transmitter/receiver (tx/rx) Matlab programs, which include phase, frequency and time recovery. The source code of the GC transceiver [13] is available online for the interested community [14].

In this paper we specifically transfer electromyography (EMG) data in both ex-vivo and in-vivo tissue employing the GC testbed [13].

\section{A. Contributions}

The main contributions of the paper are that:

- a specific electromyography (EMG) application is defined, where signals sensed from a healthy muscle are transmitted to a close one that is unable to receive natural input signals due to a nerve compression;

- a preliminary analysis is conducted to verify the possibility to transfer EMG data through GC intra-body communication by evaluating the bandwidth of both EMG signals and GC technique; 

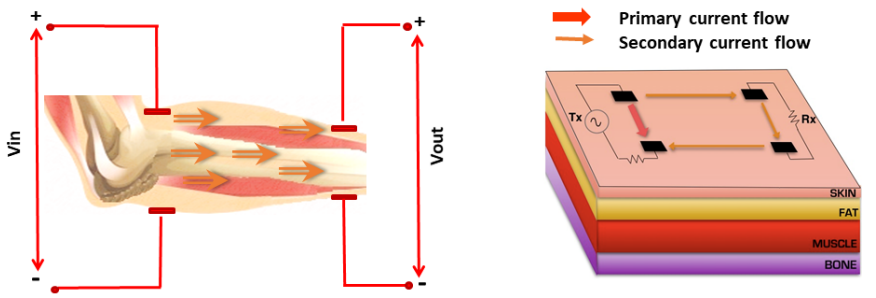

Fig. 1: GC setup on skin surface with detail on multiple tissues

- EMG data are acquired and recorded in real scenario employing needle electrodes inserted in the muscle of a person's forearm during muscle contraction;

- extensive experiments are conducted to transfer the acquired real EMG data via GC intra-body link in both ex-vivo chicken and in-vivo human tissue under different environmental conditions. The significance of this experimental study is that we are able to achieve almost error free communication, hence a reliable and robust communication, which is an important result in medical applications [15].

\section{BACKGROUND ON GALVANIC COUPLING TECHNOLOGY}

Research works on the electromagnetic waves' propagation within the body have been conducted. The studies shows that behavior depends on the used frequencies, indeed, the high frequency of RF signals turns out in high signal absorption hence and also their propagation outside the body. On the contrary, the low frequencies of GC technology allow the signals to be confined inside the body. Thus, GC does not exhibit privacy issues with the benefit of requiring two orders of magnitude less energy than RF based solutions since no energy is wasted by spreading it outside the body [7], [16].

Fig. 1 illustrates the GC transceiver where a pair of electrodes representing the transmitter are placed on the skin with a typical distance of $5 \mathrm{~cm}$ to inject weak electrical currents as data signals [13]. While the primary current flows between the two transmitter electrodes, very low secondary currents carry the information to a distant pair of receiver electrodes through multiple tissues conduction. On-skin electrodes placement is depicted in Fig. 1, although they could be placed in any tissue. The international commission on non-ionizing radiation protection (ICNIRP) recommendation [17] sets $1 \mathrm{~mA}$ as safe bound value for the current, which is easily reached by GC technology that injects currents with an amplitude around 0.5 $\mathrm{mA}$ [3].

Experimental research works verify that weak secondary currents can be detected at the receiver placed at a distance of 20-30 cm from the transmitter. The usual GC electrodes' size is around $1 \mathrm{~cm} \mathrm{[18]} \mathrm{and} \mathrm{consumes} 0.24 \mathrm{~nJ}$ per received bit, much lower than $106 \mathrm{~nJ} / \mathrm{b}$ of Zigbee [1]. This is due to the low frequencies used by GC, which make the signal restricted to the body resulting in very energy efficient communication. The tissue heating is low since the attenuation is low at the used frequencies. The GC operative frequency range $[1 \mathrm{KHz}$ - $100 \mathrm{MHz}$ [ [6] is a trade-off among tissue attenuation and interference with other natural signals.

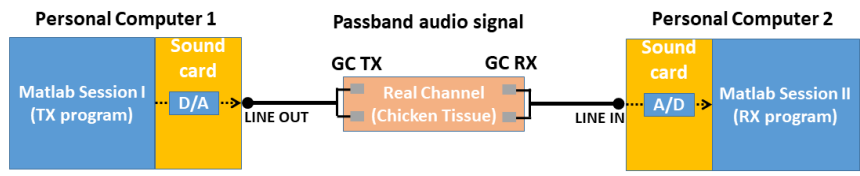

Fig. 2: Setup of the GC audio-band testbed using two PCs

\section{GC TEST SYSTEM ARCHITECTURE}

The employed testbed manages all the aspects of the communication, from signal generation to raised cosine filtering among the others [13].

\section{A. GC Testbed Overview}

The used GC testbed requires limited hardware equipment since employs only two PCs with sound card and the Matlab software to implement the transmitter and the receiver [13]. We employ battery powered PCs without connection to the grid to isolate the common ground return paths between the transmitter and the receiver as required from GC technology [17].

As shown in Fig. 2 [13], two Matlab sessions are required, one per PC, to implement the transmitter and receiver, respectively, and the sound cards are used to support real signal transmission/reception in a subset frequency range of GC technology [13]. After acquiring and recording EMG data, they are quantized and transmitted using Matlab software, then converted from digital to analog domain in order to be sent over the sound card of the transmitter. A cable is connected to the LINE OUT jack to carry the signal outside the PC. This cable is connected to two electrodes representing the GC transmitter, which send the signal over the biological tissue. At the receiver side, the two receiver's electrodes detect the received signal and send it to the other PC through a cable connected to the LINE IN jack. Then, the data are processed in the Matlab session II where the receiver program is running.

Table I shows the values of the main system's parameters. The sound card's setting include audio frequency sampling equal to $48 \mathrm{KHz}$ and 16 bits per sample. The audio parameter setting may be varied from the PC control panel, while other parameters, such as preamble length and roll-off transceiver (TRX) filters, may be changed in the tx/rx Matlab programs.

In the following, the PHY methods implemented in the transmitter and in the receiver are detailed.

\section{B. Blocks of the GC transmitter}

The EMG signal $x_{E M G}$ to be transmitted is first quantized to obtain data in digital format, then a preamble is inserted. A binary phase-shift keying (BPSK) modulation is emloyed obtaining a sequence $c_{t x}=c_{t x_{p r e}}+c_{t x_{\text {data }}}$. We use low order modulation scheme, such as BPSK, since in intra-body communication low power solutions are preferable to large bandwidth methods [3].

The sequence $c_{t x}$ is oversampled by 16 , as specified in Table I, and it is sent to a squared-root-raised-cosine (SRRC) filter. The corresponding baseband samples $x\left(n T_{s}\right)$ are thus generated, with $n=0,1, \ldots, f_{s} N-1$ and sampling time $T_{s}$. 
TABLE I: Parameters setting

\begin{tabular}{|l|c|}
\hline Parameter & Value \\
\hline Carrier frequency $f_{c}(\mathrm{KHz})$ & 10 \\
Waveform sampling frequency $f_{s_{a}}(\mathrm{KHz})$ & 48 \\
Oversampling frequency $f_{s}$ (\# of samples) & 16 \\
Sampling time $T_{s}(\mathrm{~ms})$ & 0.66 \\
RX oversampling frequency $f_{s_{r x}}$ (\# of samples) & 2 \\
roll-off of TRX filters $R$ & 0.2 \\
delay of TRX filters $D$ (\# of samples) & 8 \\
QAM modulation order $M$ & 2 \\
RX Wiener filter length $N_{f}$ (\# of samples) & 11 \\
Modulated sequence $N$ (\# of symbols) & 10000 \\
Preamble length $N_{\text {pre }}$ (\# of symbols) & 1000 \\
\hline
\end{tabular}

Then, the resulting sequence is upconverted to the carrier frequency by multiplying it by the cos signal that represents the local oscillator (LO) obtained via software-define radio in Matlab program. An audio passband transmitted signal $s_{t x}$ is thus generated:

$$
s_{t x}\left(n T_{s_{a}}\right)=x\left(n T_{s_{a}}\right) \cos \left(2 \pi f_{c} n T_{s_{a}}\right)
$$

where $T_{s_{a}}=1 / f_{s_{a}}, f_{s_{a}}=48 \mathrm{KHz}$ and $f_{c}$ is the carrier frequency. According to Table I, $T_{s}=32 T_{s_{a}}$ with a net data rate $R=1.5 \mathrm{kbs}$.

The obtained audible signal $s_{t x}$ passes through the sound card's internal amplifier, which is then sent out to the LINE OUT jack by the Matlab audioplayer function, which play the software-generated transmitted signal. The output signal is then sent to two electrodes representing the GC transmitter by means of a wire (see Fig. 2 and Fig. 5).

\section{Blocks of the $G C$ receiver}

To recover the baseband signal, a local oscillator is used at the receiver with the same frequency and phase of the modulating carrier. However, phase errors may occur lowering the performance and a carrier synchronizer is thus needed [19].

The blocks of the receiver can be divided in three macroblocks: (i) signal recording and digital down-conversion, (ii) carrier frequency recovery, (iii) burst detection and fractionally spaced Wiener filter.

1) Signal recording and digital down-conversion: A Matlab session in the PC connected with the GC receiver's electrodes run the receiver program, which includes the Matlab audiorecorder function to record the received signal and store the data in the array $\mathbf{s}_{r x}$.

After recording $\mathbf{s}_{r x}$ in digital format, a digital downconversion is performed by multiplying the received sampled signal $\mathbf{s}_{r x}$ by $\cos \left(2 \pi f_{c}^{\prime} n T_{s_{a}}\right)$ and $\sin \left(2 \pi f_{c}^{\prime} n T_{s_{a}}\right)$, where $f_{c}^{\prime}$ is the carrier frequency at the receiver, thus obtaining $d^{i}\left(n T_{s_{a}}\right)$ and $d^{q}\left(n T_{s_{a}}\right)$, respectively. The sequence $d\left(n T_{s_{a}}\right)=$ $d^{i}\left(n T_{s_{a}}\right)+j d^{q}\left(n T_{s_{a}}\right)$ is sent to an SRRC filter, which results in a baseband received signal $r\left(n T_{s_{a}}\right)$ with $n=0,1, \ldots, f_{s} N-$ 1. The output $\mathbf{r}$ may encounter frequency synchronization problem.
Then, $\mathbf{r}$ is decimated by eight so that the next following blocks work at two samples per symbol $\left(f_{s_{r x}}=2\right.$ as set in Table I). The obtained sampled signal may be expressed as

$$
r\left(n T_{s}\right)=s_{t x}\left(n T_{s}\right) e^{j \theta}+v\left(n T_{s}\right)
$$

where $n=0,1, \ldots, f_{s_{r x}} N-1, s_{t x}\left(n T_{s}\right)$ is the envelope samples of the passband signal, $v\left(n T_{s}\right)$ is the sampled additive white gaussian noise (AWGN), $\theta$ is the random phase noise due to time delay.

2) Carrier frequency recovery: To overcome the frequency synchronization issue, a sawtooth frequency recovery method is implemented. The carrier frequency offset $\Delta_{f}=f_{c}-f_{c}^{\prime}$ is estimated and the received signal is contro-rotated by $2 \pi \Delta_{f}$ to compensate its effect.

The estimate $\Delta_{f}$ is calculated off-line by setting a step size $\delta_{f}$ and calculating the value $m$, with $m \in\{1, \ldots, M\}$, for which $\Delta_{f}=m \delta_{f}$ minimizes the mean squared error (MSE):

$$
\underset{m \delta_{f}}{\operatorname{minimize}} E\left(\left\|\mathbf{c}_{t x_{d a t a}}-\hat{\mathbf{c}}_{t x_{d a t a}}\right\|^{2}\right)
$$

where $\mathbf{c}_{t x_{\text {data }}}$ is the sequence at the transmitter after the modulation mapper and $\hat{\mathbf{c}}_{t x_{d a t a}}$ is the sequence at the receiver before the modulation decision. Each vector is normalized to the power, i. e., $\mathbf{c}_{t x_{d a t a}}=\mathbf{c}_{t x_{d a t a}} / \sqrt{P_{t x}}$ and $\hat{\mathbf{c}}_{t x_{d a t a}}=\hat{\mathbf{c}}_{t x_{\text {data }}} / \sqrt{P_{r x}}$ with $P_{t x}=\sum_{n=1}^{N}\left(c_{t x_{\text {data }}}(n)\right)^{2} / N$ and $P_{r x}=\sum_{n=1}^{N}\left(\hat{c}_{t x_{\text {data }}}(n)\right)^{2} / N$, where $N$ is the length of the vectors. To speed up the search, the value of $\delta_{f}$ and $M$ have been chosen experimentally according to the frequency offset values usually obtained. The minimization problem (3) is equivalent to find the value $\Delta_{f}=m \delta_{f}$ that maximizes the SNR at the receiver, defined as the inverse of the error $e=E\left(\left\|\mathbf{c}_{t x_{\text {data }}}-\hat{\mathbf{c}}_{t_{\text {data }}}\right\|^{2}\right)$, as calculated in Sec. III-D.

Once the value $\Delta_{f}$ is obtained, the signal $r\left(n T_{s}\right)$ is controrotated to compensate the effect of the frequency offset:

$$
y\left(n T_{s}\right)=r\left(n T_{s}\right) e^{-j 2 \pi \Delta_{f} n T_{s}}
$$

It is worth to note that, according to (3), $\Delta_{f}$ is calculated offline after obtaining the sequence $\hat{\mathbf{c}}_{t x_{d a t a}}$ before the modulation decision, and then the contro-rotation is performed backward in the carrier frequency recovery block.

3) Burst detection and fractionally spaced Wiener filter: A correlation technique is used for burst detection to correctly shift in time the received signal. Then the signal is equalized with a fractionally spaced Wiener filter, which also performs symbol timing thus improving the previously estimated delay. The Wiener filter also obtains a mean phase compensation, thus avoiding to employ complex phase-locked loop (PLL) or Kalman based methods [20]. Small phase errors are still present since only a mean compensation is performed but they do not degrade significantly the performance as shown in Sec. $\mathrm{V}$.

Going in more details, the packet is composed by a preamble followed by data, and the preamble is exploited in the burst detection method based on correlation. The start of the preamble is thus obtained. The transmitted preamble, assumed to be known at the receiver, is cross-correlated with the received data so that the position of the correlation's peak 
estimates the preamble start position:

$$
\hat{t}_{p}=\arg \max _{n}\left|\sum_{k=0}^{L-1} y\left((n+k) T_{s}\right) p^{*}\left(k T_{s}\right)\right|
$$

in which $\hat{t}_{p}$ is the estimated sample index where the reference preamble $p$ begins, $N_{\text {pre }}$ is the length of the preamble, $L=f_{s_{r x}} N_{\text {pre }}$ (oversampling frequency $f_{s_{r x}}=2$ ) since the receiver works at two samples per symbol, and $n=$ $0,1, \ldots, f_{s_{r x}} N-1$.

Hence, the received signal is shifted in time according to the estimated preamble's start $\hat{t}_{p}$ and is equalized with a Wiener filter, which is also able to perform symbol timing thus improving the delay estimated with (5).

The coefficients $w(i)$ of the Wiener filter, with $i=$ $1, \ldots, N_{f}$, are obtained by using the shifted received preamble $y_{p}\left(n T_{s}\right)=y\left(n T_{s}+\hat{t}_{p}\right)$ and the known transmitted one $p\left(n T_{s}\right)$ with $n=0,1, \ldots, f_{s_{r x}} N_{p r e}-1$. Specifically, the vector $\mathbf{w}=\left[w_{1}, w_{2}, \ldots, w_{N_{f}}\right]$ of the coefficients are calculated by minimizing the MSE between the transmitted and estimated preamble:

$$
\epsilon=E\left\{\left[p\left(n T_{s}\right)-\hat{p}\left(n T_{s}\right)\right]^{2}\right\} .
$$

Setting the partial derivatives of the error (6) equal to zero

$$
\frac{\partial \epsilon}{\partial w_{i}}=0, \text { for } i=1,2, \ldots, N_{f}
$$

it is possible to solve (6), (7) for the coefficients $w_{i}$ by inverting an autocorrelation matrix of size $N_{f} \times N_{f}$, leading to the Wiener-Hopf equation

$$
\mathbf{w}=\mathbf{A}_{y_{p}}^{-1} \mathbf{a}_{p y_{p}}
$$

where $\mathbf{A}_{y_{p}}=\left\{a_{y_{p}}(k)\right\}$ is the autocorrelation matrix of the received preamble $y_{p}$, whose elements are $a_{y_{p}}(k)=$ $E\left\{y_{p}(i-k) y_{p}(i)\right\} . \mathbf{a}_{p y_{p}}=\left\{a_{p y_{p}}(k)\right\}$ is the cross-correlation vector between the transmitter preamble $p$ and the received one $y_{p}$, whose elements are defined as $a_{p y_{p}}(k)=$ $E\left\{p(i-k) y_{p_{k}}(i)\right\}$, and $i, k=1,2, \ldots, f_{s_{r x}} N_{f}$.

The Wiener filter is applied to all the sequence so that its output is the estimated transmitted sequence $\hat{c}_{t x}\left(n T_{s}\right)$ :

$$
\hat{c}_{t x}\left(n T_{s}\right)=\sum_{l=0}^{N_{f}-1} w\left(l T_{s}\right) y\left((n-l) T_{s}+\hat{t}_{p}\right)
$$

After a down-sampling operation at one sample per symbol, the BPSK decision is performed using $\operatorname{Re}\left(\hat{c}_{t x}\left(n T_{s}\right)\right)$ so that the symbol sequence is demapped to a bit sequence. Then, the preamble is removed, and the procedure inverse to the quantization at the transmitter is performed to finally reconstruct the original EMG signal at the receiver.

\section{Equivalent Signal to Noise Ratio}

We here calculate the SNR at the receiver as in [13], which is employed in the frequency carrier recovery (see Sec. III-C2) and as performance metric alternative to bit error rate (BER) to avoid too extensive experiments since almost error free results are achieved.

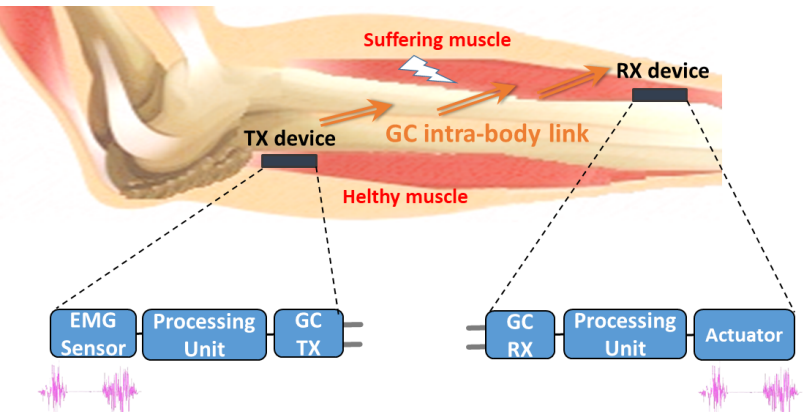

Fig. 3: Conceptual framework of intra-body GC link for a muscle suffering nerve compression

The error $e$ is first defined as MSE:

$$
e=E\left(\left\|\frac{\mathbf{c}_{t x_{\text {data }}}}{\sqrt{P_{t x}}}-\frac{\operatorname{Re}\left(\hat{\mathbf{c}}_{t x_{\text {data }}}\right)}{\sqrt{P_{r x}}}\right\|^{2}\right),
$$

in which $\mathbf{c}_{t x_{\text {data }}}$ is the sequence at the transmitter after the BPSK mapper, $\hat{\mathbf{c}}_{t x_{\text {data }}}$ is the sequence at the receiver before the BPSK decision, that has been downsampled at one bit per symbol. The sequences at the transmitter and receiver are then normalized to the power $P_{t x}$ and $P_{r x}$, where $P_{t x}=\sum_{n=1}^{N}\left(c_{t x_{\text {data }}}(n)\right)^{2} / N$ and $P_{r x}=$ $\sum_{n=1}^{N}\left(\operatorname{Re}\left(\hat{c}_{t x_{\text {data }}}(n)\right)\right)^{2} / N$. $S N R$ is hence defined as the inverse of the error $e$ in (10).

Since the GC intra-body channel can be approximated as AWGN [21], the probability of error $P_{b}$ may be calculated as $P_{b}=\frac{1}{2} \operatorname{erfc}\left(\sqrt{\left(E_{b} / N_{0}\right)}\right)$, in which $\left(E_{b} / N_{0}\right)$ is the energyper-bit to noise ratio corresponding to the SNR for BPSK.

\section{EMG DATA TRANSFER}

In this paper we specifically focus on EMG data transmission, which could be useful in some medical applications as specified in the following.

\section{A. Application Scenario}

We have conducted a preliminary evaluation to apply the GC test system to a scenario where a muscle can not receive the natural input signal for contraction due to nerve injury and/or compression [13]. During a muscular contraction some different muscles are involved, hence a possible solution may consist in exploiting the signal on a healthy muscle and use it on a muscle that is unable to receive the natural signal since its dedicated nerve suffers a compression. Thus, EMG signals acquired from an healthy muscle are used as input signal to trigger the muscle suffering nerve compression. An intrabody GC communication link may be established between a device placed on the healthy muscle, which senses the EMG signal and transmits it to a receiver device placed on the muscle suffering nerve compression. Here the device detects and processes the signal and use it as input to trigger the muscle. Fig. 3 shows the system configuration of the proposed application.

For the feasibility of the proposed approach, we have analyzed the required EMG bandwidth with the allowed GC 


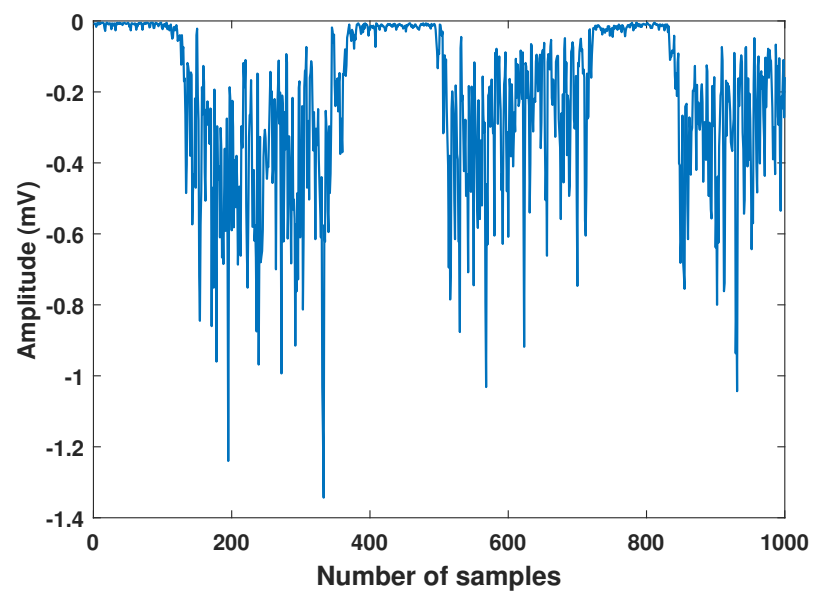

Fig. 4: EMG signal acquired with needle electrodes during forearm's muscle contraction

frequency range and carrier of the used GC audio band-based testbed [13]. EMG is a narrow band signal with a bandwidth of [12-500] Hz, thus it is possible to send EMG signals over the used testbed with the setting in Table I. The sampling frequency $\left(f_{s_{a}}=48 \mathrm{KHz}\right)$ is higher enough than the frequency carrier $\left(f_{c}=10 \mathrm{KHz}\right)$ to modulate the EMG signal.

\section{B. EMG data acquisition}

EMG signals have been acquired and recorded as real data to be sent via a GC intra-body communication link. Needle electrodes have been inserted in the muscle of a person's forearm during muscle contraction, whose recorded EMG signal is shown in Fig. 4. The signal has been recorded for $10 \mathrm{~s}$ with a sampling frequency of $20 \mathrm{KHz}$, then 1000 mean values has been extracted and recorded with a consequent net sampling rate of $100 \mathrm{~Hz}$. This value, according to the bandwidth calculation in Sec. IV-A, allows to transmit the EMG signal via the GC sound card-based test system. The amplitude of the EMG signal is expressed in $\mathrm{mV}$ as shown in Fig. 4.

\section{EMG data transmission}

To transmit the EMG signal, its values are first quantized and then transmitted with a BPSK modulation. The number of bit for the quantization has be chosen equal to 8 , which allows to achieve a MSE between the reconstructed quantized data at the receiver and the original ones in the order of $10^{-5}$, as specified in Sec. V. The error $e_{E M G}$ is defined as MSE:

$$
e_{E M G}=E\left(\left\|\frac{\mathbf{x}_{E M G}}{\sqrt{P_{\mathbf{x}_{E M G}}}}-\frac{\hat{\mathbf{x}}_{E M G}}{\sqrt{P_{\hat{\mathbf{x}}_{E M G}}}}\right\|^{2}\right)
$$

where $\mathbf{x}_{E M G}$ is the sequence of the acquired EMG data before being quantized and transmitted, $\hat{\mathbf{x}}_{E M G}$ is the sequence of the reconstruted EMG data at the receiver. The sequences before the transmission and after the reconstruction at the receiver are normalized to the power $P_{\mathbf{x}_{E M G}}$ and $P_{\hat{\mathbf{x}}_{E M G}}$, where $P_{\mathbf{x}_{E M G}}=$ $\sum_{n=1}^{N}\left(x_{E M G}(n)\right)^{2} / N$ and $P_{\hat{\mathbf{x}}_{E M G}}=\sum_{n=1}^{N}\left(\hat{x}_{E M G}(n)\right)^{2} / N$.

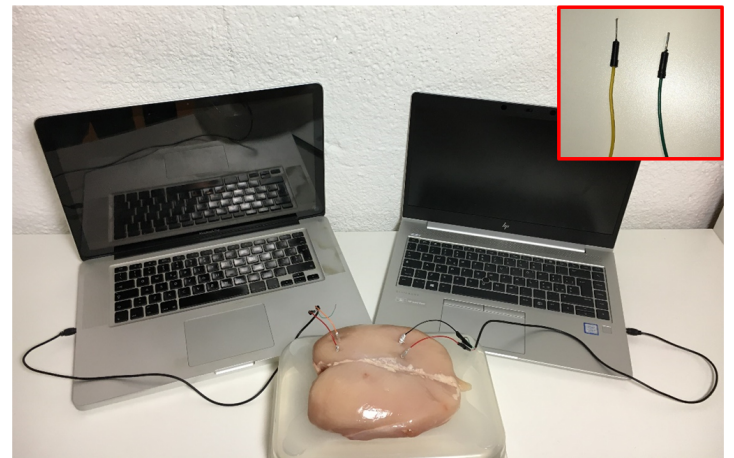

Fig. 5: GC experimental setup on ex-vivo tissue

\section{EXPERIMENTAL SETUP AND RESULTS}

In the following, the experimental environment is described and the obtained results are illustrated in both ex-vivo and in-vivo configuration.

\section{A. Experimental environment}

We consider two configurations: one with GC electrodes implanted in ex-vivo chicken tissue (Fig. 5) and another one with wearable GC electrodes placed on in-vivo skin (Fig. 6).

For the case of ex-vivo tissue we employ low cost regular leads, covered with aluminum foil, as shown in Fig. 5, to avoid oxidation due to the water content of the chicken breast. We use really small size circle electrodes (in the order of 0.5 $\mathrm{mm}$ diameter) to test a real configuration scenario for future miniaturized medical devices. For the case of in-vivo tests we employ commercial electrodes with $3 \mathrm{~cm}$ or $1 \mathrm{~cm}$ size placed on the leg's skin as shown in Fig. 6.

The parameters setting is detailed in Table I, a random generated sequence is used for the preamble and the performance is calculated over 100 runs. The GC link transmission is evaluated on a $21 \mathrm{~cm} \times 16 \mathrm{~cm} \times 6 \mathrm{~cm}$ chicken breast for the ex-vivo scenario and on a leg for the in-vivo case.

The transmission power $P_{t x}$ is in the order of $10 \mu \mathrm{W}$ and the inter-distance between the electrodes at both the transmitter and receiver is set equal to $1.5 \mathrm{~cm}$ for the ex-vivo tests and $6 \mathrm{~cm}$ for in-vivo experiments if otherwise stated, while the distance between the transmitter and receiver is varied during the experiments.

\section{B. Evaluation of EMG data transfer over ex-vivo tissue}

First, a qualitative analysis of the established GC intra-body link is conducted showing the transmitted and received signal in time and frequency domain, as illustrated in Fig. 7. Then, a quantitative investigation is carried out in terms of equivalent SNR calculated as in Sec. III-D (see Table II).

Fig. 7 shows the transmitted and received signals with a inter-electrodes distance equal to $1.5 \mathrm{~cm}$ at both transmitter and receiver, and the distance between transmitter and receiver equal to $11 \mathrm{~cm}$. Both time and frequency domain are considered, and the received signal is represented at various steps showing the distortion and compensation at the receiver. The 


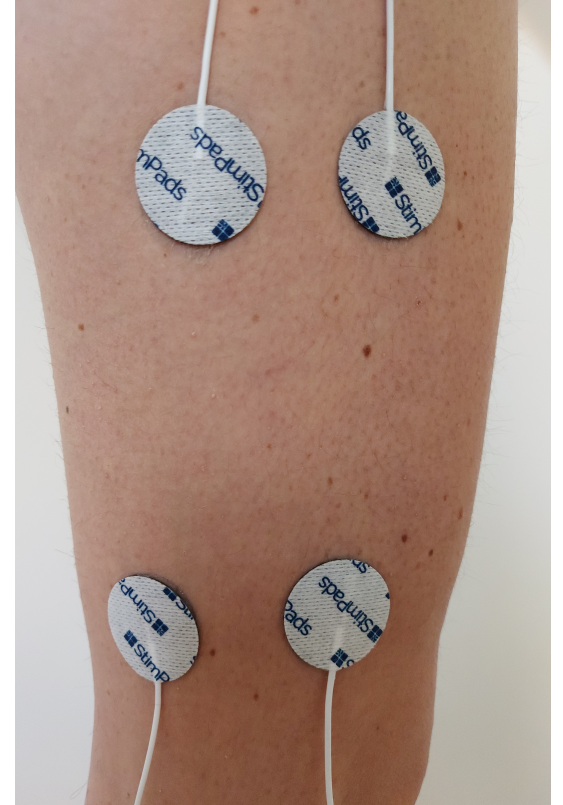

Fig. 6: GC experimental setup on in-vivo skin surface

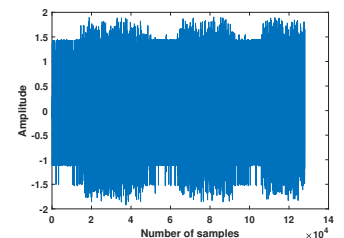

(a) Tx signal in time domain

(c) Rx signal in time domain
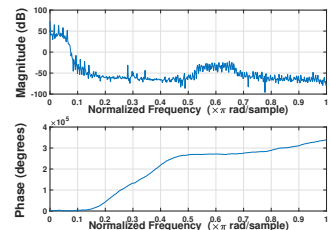

(e) Rx signal after filter in freq domain
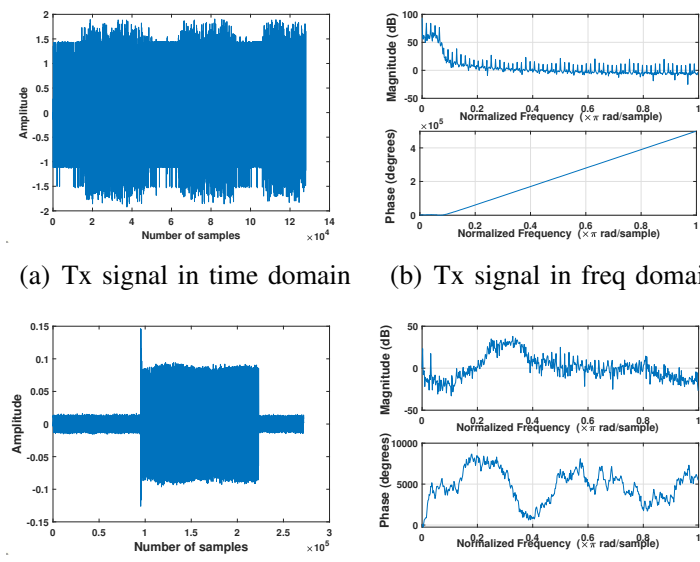

(b) Tx signal in freq domain

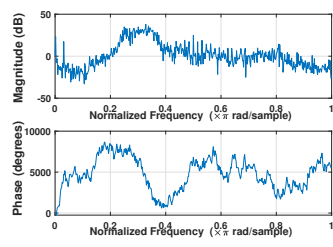

(d) Rx signal in freq domain

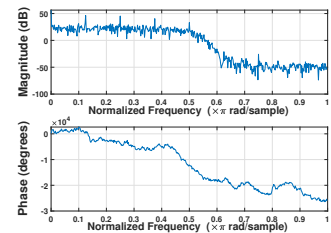

(f) Rx signal after filter and freq offset compensation in freq domain

Fig. 7: Transmitted and received signal with BPSK modulation in ex-vivo tissue (distance $d_{t x-r x}=11 \mathrm{~cm}$ )

amplitude of the received signal shows an order of magnitude less than the transmitted one, comparing Fig. 7(a) and Fig. 7(c).

Since we achieve almost error free performance, too extensive experiments would be required for reliable BER values, thus we evaluate the equivalent SNR, calculated as in Sec. III-D. Table II illustrates the obtained SNR while varying the
TABLE II: Performance in ex-vivo tissue

\begin{tabular}{|c|c|c|}
\hline Distance $_{\mathbf{t x}-\mathbf{r x}}(\mathbf{c m})$ & $\mathbf{M S E}_{\mathbf{b i t}}$ & $\mathbf{S N R}(\mathbf{d B})$ \\
\hline 2 & 0.0095 & 20.21 \\
6 & 0.0099 & 20.04 \\
11 & 0.0117 & 19.32 \\
\hline
\end{tabular}

TABLE III: Performance in in-vivo tissue

\begin{tabular}{|c|c|c|}
\hline Distance $_{\text {tx }-\mathbf{r x}}(\mathbf{c m})$ & $\mathbf{M S E}_{\text {bit }}$ & $\mathbf{S N R}(\mathbf{d B})$ \\
\hline 9 & 0.0090 & 20.45 \\
17 & 0.0095 & 20.23 \\
25 & 0.0098 & 20.08 \\
\hline
\end{tabular}

distance between transmitter and receiver. The table shows that the performance remains high in all the configurations, with almost $1 \mathrm{~dB}$ lower SNR for longer distance equal to $11 \mathrm{~cm}$. The normalized MSE between the EMG signal to be transmitted and the reconstructed one at the receiver, calculated as in (11), is in the order of $10^{-5}$. The mean estimated frequency offset over 100 runs is around $2 \mathrm{~Hz}$. Future works include testing longer distances and lower transmitted power to evaluate the performance limits of the developed system.

Fig. 8 illustrates the comparison between the EMG signal and its reconstructed version after being transferred via a GC intra-body link. The figure shows that the reconstructed signal is almost equal to the original one.

\section{Evaluation of EMG data transfer over in-vivo tissue}

Also in-vivo tests show almost error free performance. Although the skin tissue is less conductive of the muscle one, the obtained high performance is comparable to the one achieved in ex-vivo chicken breast since we employ larger electrodes $(3 \mathrm{~cm}$ diameter as in Fig. 9 and Table III or $1 \mathrm{~cm}$ as in Fig. 10). When the GC electrodes are placed on the skin, the weak currents flow not only over the skin but within the heterogeneous tissues reaching also the muscle. The low currents flowing in the human tissues are imperceptible to the person under testing.

Fig. 9 illustrates the transmitted and received signal after in-vivo transmission both in time and frequency domain at different steps. Table III shows the performance in terms of equivalent SNR calculated as in Sec. III-D while varying the distance between the GC transmitter and receiver. The table reveals that the performance are very good and stable while varying the distance. This is due to the large size of $3 \mathrm{~cm}$ electrodes employed. Indeed, Fig. 10, where $1 \mathrm{~cm}$ electrodes are used, illustrates that the reconstructed EMG signals shows some little differences compared to the original one. Future works include extensive experiments with different electrode sizes and distances between the transmitter and the receiver.

\section{CONCLUSION}

We have conducted extensive experimental tests to transfer real EMG data via GC intra-body link. First, the employed GC system has been described and then the application scenario has been detailed. After a preliminary analysis to confirm 


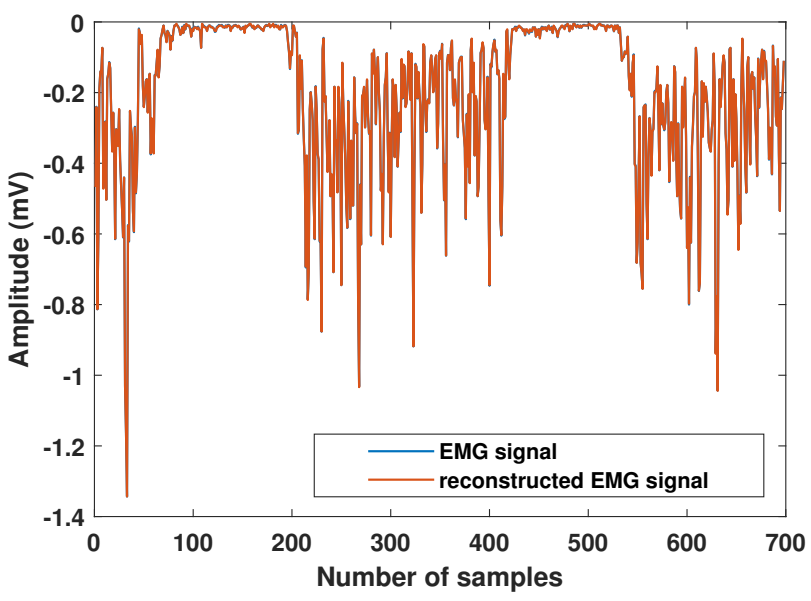

(a) EMG signal vs the reconstructed EMG one

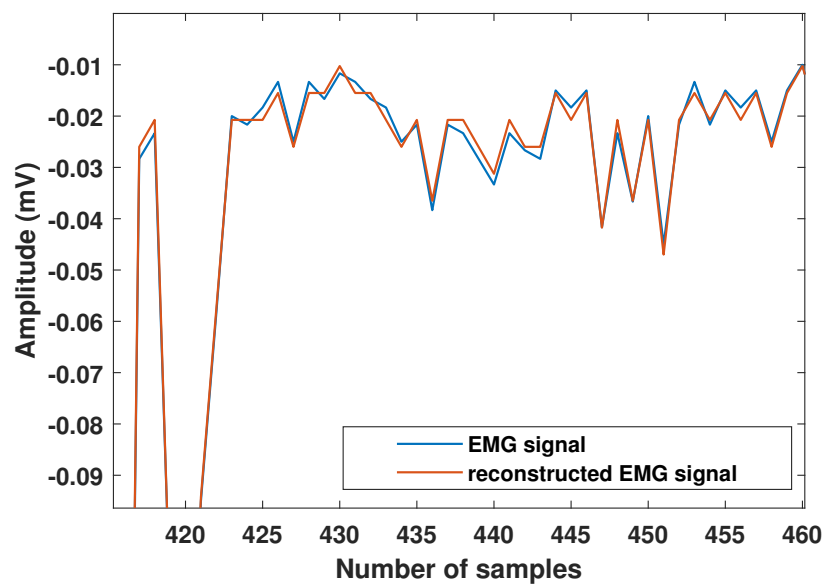

(b) Magnifying version of the figure 8(a) to show the differences between the signals

Fig. 8: Comparison between the EMG signal and its reconstructed version after transmission in ex-vivo tissue (distance $d_{t x-r x}=11 \mathrm{~cm}$ and $0.5 \mathrm{~mm}$ electrodes)

the possibility to transfer EMG data through GC intra-body communication by evaluating the bandwidth of both EMG signals and GC technology, real intra-body communication for EMG data transfer has been tested in both ex-vivo chicken and in-vivo human tissue. The main achievement of this experimental study is that we are able to obtain almost error free performance, hence a robust and reliable communication, which is of primary importance in medical applications.

Future works include experiments to evaluate the effect of carrier frequency, inter-electrodes distance and electrodes placement, among the others. Also, multiple implants scenario will be evaluated, in which it is essential to develop opportunistic wake-up methods and devices' localization [22]-[24], or to use resource allocation solutions [25], [26] to minimize the energy consumption, as required in intra-body networks.

\section{REFERENCES}

[1] M. Seyedi, B. Kibret, D.T.H. Lai and M. Faulkner, "A Survey on Intrabody Communications for Body Area Network Applications," IEEE

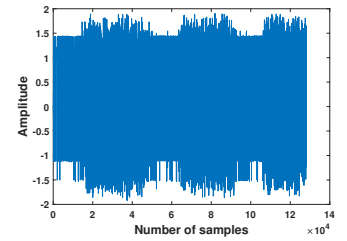

(a) Tx signal in time domain

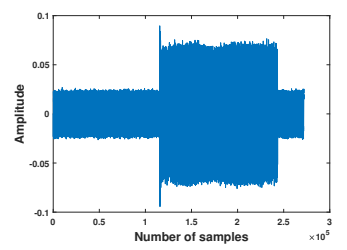

(c) Rx signal in time domain

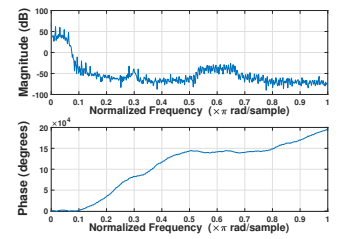

(e) $\mathrm{Rx}$ signal after filter in freq domain

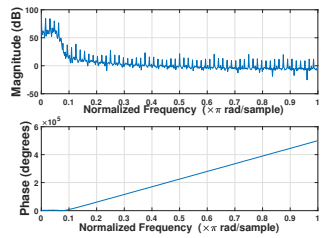

(b) Tx signal in freq domain

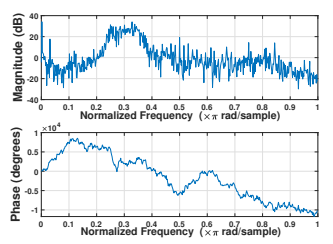

(d) Rx signal in freq domain

(f) Rx signal after filter and frec offset compensation in freq domain

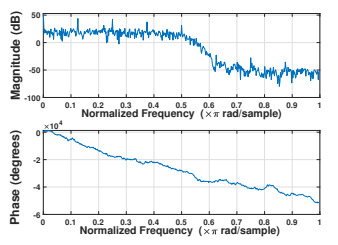

Fig. 9: Transmitted and received signal with BPSK modulation in in-vivo tissue (distance $d_{t x-r x}=25 \mathrm{~cm}$ and $3 \mathrm{~cm}$ electrodes)

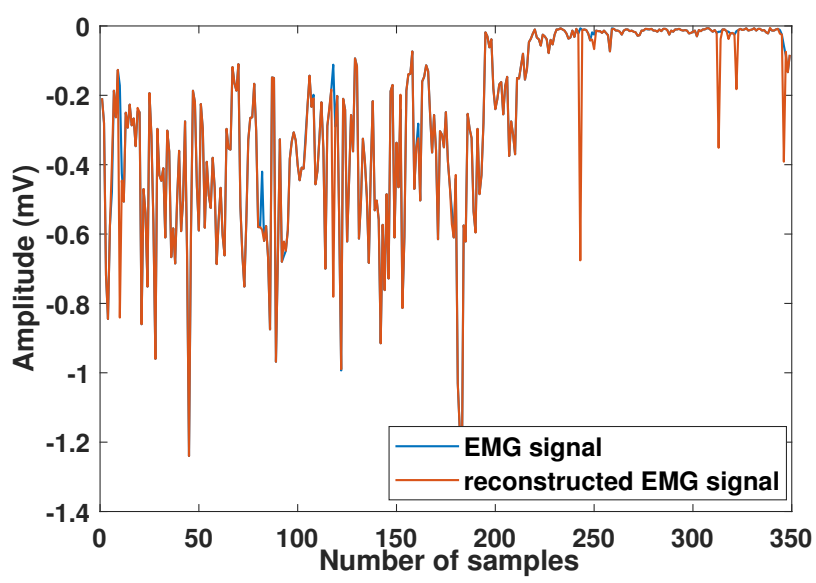

Fig. 10: Comparison between EMG signal and its reconstructed version after in-vivo transmission. The configuration setting consists in electrodes places on skin, electrodes size equal to $1 \mathrm{~cm}$, inter-electrodes distance equal to $4 \mathrm{~cm}$ and distance $d_{t x-r x}=20 \mathrm{~cm}$ 
Trans. Biomedical Engineering vol. 60, n.8, pp. 2067-2079, Aug. 2013, doi: 10.1109/TBME.2013.2254714.

[2] B. Latré, B. Braem, I. Moerman et al., "A survey on wireless body area networks," Wireless Netw 17, 1-18, 2011.

[3] W. J. Tomlinson, S. Banou, C. Yu, M. Stojanovic and K. R. Chowdhury, "Comprehensive Survey of Galvanic Coupling and Alternative IntraBody Communication Technologies," in IEEE Communications Surveys \& Tutorials, vol. 21, no. 2, pp. 1145-1164, Secondquarter 2019, doi: 10.1109/COMST.2018.2879643.

[4] L. Galluccio, T. Melodia, S. Palazzo and G. E. Santagati, "Challenges and Implications of Using Ultrasonic Communications in Intra-body Area Networks," in proc. of IEEE Wireless On-demand Network Systems and Services, Courmayeur, Italy, Jan. 2012.

[5] M. S. Wegmueller et al., "Galvanic Coupling Enabling Wireless Implant Communications," in IEEE Transactions on Instrumentation and Measurement, vol. 58, no. 8, pp. 2618-2625, Aug. 2009, doi: 10.1109/TIM.2009.2015639.

[6] M. A. Callejón, J. Reina-Tosina, D. Naranjo-Hernández and L. M. Roa, "Galvanic Coupling Transmission in Intrabody Communication: A Finite Element Approach," in IEEE Transactions on Biomedical Engineering, vol. 61, no. 3, pp. 775-783, March 2014, doi: 10.1109/TBME.2013.2289946.

[7] M. Swaminathan, F. S. Cabrera, J. S. Pujol, U. Muncuk, G. Schirner and K. R. Chowdhury, "Multi-Path Model and Sensitivity Analysis for Galvanic Coupled Intra-Body Communication Through Layered Tissue," in IEEE Transactions on Biomedical Circuits and Systems, vol. 10, no. 2, pp. 339-351, April 2016, doi: 10.1109/TBCAS.2015.2412548.

[8] M. S. Wegmueller, M. Oberle, N. Felber, N. Kuster and W. Fichtner, "Signal Transmission by Galvanic Coupling Through the Human Body," in IEEE Transactions on Instrumentation and Measurement, vol. 59, no. 4, pp. 963-969, April 2010, doi: 10.1109/TIM.2009.2031449.

[9] M. J. Seyedi and D. Lai, "A Novel Intrabody Communication Transceiver for Biomedical Applications," Springer, Singapore, 2017.

[10] W. J. Tomlinson, K. R. Chowdhury and C. Yu, "Galvanic coupling Intra-Body Communication link for real-time channel assessment," 2016 IEEE Conference on Computer Communications Workshops (INFOCOM WKSHPS), San Francisco, CA, 2016, pp. 968-969, doi: 10.1109/INFCOMW.2016.7562220.

[11] R. Noormohammadi, A. Khaleghi and I. Balasingham, "Galvanic Impulse Wireless Communication for Biomedical Implants," in IEEE Access, vol. 9, pp. 38602-38610, 2021, doi: 10.1109/ACCESS.2021.3064206.

[12] A. Vizziello, P. Savazzi, F. Kulsoom, G. Magenes and P. Gamba, "A Novel Galvanic Coupling Testbed Based on PC Sound Card for Intrabody Communication Links," In: Mucchi L., Hämäläinen M., Jayousi S., Morosi S. (eds) Body Area Networks: Smart IoT and Big Data for Intelligent Health Management. BODYNETS 2019. Lecture Notes of the Institute for Computer Sciences, Social Informatics and Telecommunications Engineering, vol 297. Springer, Cham.

[13] A. Vizziello, P. Savazzi, G. Magenes and P. Gamba, "PHY Design and Implementation of a Galvanic Coupling Testbed for Intra-Body Communication Links," in IEEE Access, vol. 8, pp. 184585-184597, 2020, doi: 10.1109/ACCESS.2020.3029862.

[14] A. Vizziello, and P. Savazzi "Code of Sound Card-based Testbed for an Intra-body Galvanic Coupling link," [Source Code], 2020, https://doi.org/10.24433/CO.7879297.v1

[15] W. K. Chen et al., "Design of Galvanic Coupling Intra-Body Communication Transceiver Using Direct Sequence Spread Spectrum Technology," in IEEE Access, vol. 8, pp. 84123-84133, 2020, doi: 10.1109/ACCESS.2020.2991206.

[16] M. Swaminathan, A. Vizziello, D. Duong, P. Savazzi and K. R. Chowdhury, "Beamforming in the body: Energy-efficient and collisionfree communication for implants," IEEE INFOCOM 2017 - IEEE Conference on Computer Communications, Atlanta, GA, 2017, pp. 1-9, doi: 10.1109/INFOCOM.2017.8056989.

[17] S. Banou et al., "Beamforming Galvanic Coupling Signals for IoMT Implant-to-Relay Communication," in IEEE Sensors Journal, vol. 19, no. 19, pp. 8487-8501, 1 Oct.1, 2019, doi: 10.1109/JSEN.2018.2886561.

[18] M. Li et al. "The Modeling and Simulation of the Galvanic Coupling Intra-Body Communication via Handshake Channel." Sensors (Basel, Switzerland) vol. 17,4 863. 14 Apr. 2017, doi:10.3390/s17040863.

[19] U. Mengali, "Synchronization Techniques for Digital Receivers," Springer, 1997, doi: 10.1007/978-1-4899-1807-9

[20] A. Vizziello, P. Savazzi and R. Borra, "Joint Phase Recovery for XPIC System Exploiting Adaptive Kalman Filtering," in IEEE Communications Letters, vol. 20, no. 5, pp. 922-925, May 2016, doi: 10.1109/LCOMM.2016.2542798.
[21] W. J. Tomlinson, F. Abarca, K. R. Chowdhury, M. Stojanovic and C. Yu, "Experimental assessment of human-body-like tissue as a communication channel for galvanic coupling," 2015 IEEE 12th International Conference on Wearable and Implantable Body Sensor Networks (BSN), Cambridge, MA, 2015, pp. 1-6, doi: 10.1109/BSN.2015.7299424.

[22] S. Kianoush, A. Vizziello and P. Gamba, "Energy-Efficient and MobileAided Cooperative Localization in Cognitive Radio Networks," in IEEE Transactions on Vehicular Technology, vol. 65, no. 5, pp. 3450-3461, May 2016, doi: 10.1109/TVT.2015.2441733.

[23] A. Vizziello, S. Kianoush, L. Favalli and P. Gamba, "Location based routing protocol exploiting heterogeneous primary users in cognitive radio networks," 2013 IEEE International Conference on Communications (ICC), Budapest, 2013, pp. 2890-2894, doi: 10.1109/ICC.2013.6654980.

[24] M. Stelzner and T. Immo, "FCNN: Location Awareness Based on a Lightweight Hop Count Routing Body Coordinates Concept," in Proc. of the Sixth Annual ACM International Conference on Nanoscale Computing and Communication, 2019.

[25] A. Vizziello, I. F. Akyildiz, R. Agusti, L. Favalli and P. Savazzi, "Cognitive Radio Resource Management Exploiting Heterogeneous Primary Users," 2011 IEEE Global Telecommunications Conference - GLOBECOM 2011, Houston, TX, USA, 2011, pp. 1-5, doi: 10.1109/GLOCOM.2011.6134378

[26] Vizziello, A., Akyildiz, I.F., Agustí, R. et al. Cognitive radio resource management exploiting heterogeneous primary users and a radio environment map database. Wireless Netw 19, 1203-1216 (2013). https://doi.org/10.1007/s11276-012-0528-y

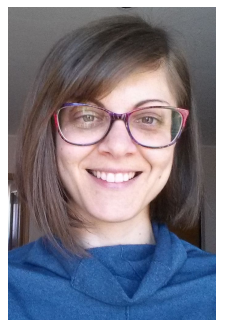

Anna Vizziello (M'09-SM'20) received the Laurea degree in electronic engineering and the Ph.D. degree in electronics and computer science from the University of Pavia, Italy, in 2007 and in 2011, respectively.

She is currently a Research Fellow in the Telecommunication \& Remote Sensing Laboratory at the University of Pavia, Italy. From 2007 to 2009 she also collaborated with European Centre for Training and Research in Earthquake Engineering (EUCENTRE) working in the Telecommunications and Remote Sensing group. From 2009 to 2010 she has been a visiting researcher at Broadband Wireless Networking Lab at Georgia Institute of Technology, Atlanta, GA, in summer 2009 and 2010 at Universitat Politècnica de Catalunya, Barcelona, Spain, and in winter 2011 and in summer 2016 at Northeastern University, Boston MA. Her research interests are Intra-Body networks, 5G Radio Technologies, Cognitive Radio Networks, and Wireless Sensor Networks.

Dr. Vizziello has been included in the 2018 list of "N2Women: Rising Stars in Computer Networking and Communications" for outstanding and impactful contributions in the area of networking/communications, supported by IEEE Communication Society.

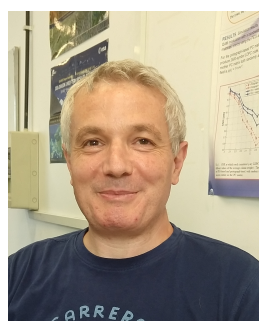

Pietro Savazzi (M'96-SM'17) received the Laurea degree in electronics engineering and the Ph.D. degree in electronics and computer science from the University of Pavia, Italy, in 1995 and in 1999, respectively.

In 1999, he joined Ericsson Lab Italy, in Milan, as a system designer, working on broadband microwave systems. In 2001 he moved to Marconi Mobile, Genoa, Italy, as a system designer in the filed of 3G wireless systems. Since 2003 he has been working at the University of Pavia where he is currently teaching, as an assistant professor, two courses on signal processing and wireless sensor networks. His main research interests are in wireless communication and sensor systems. 


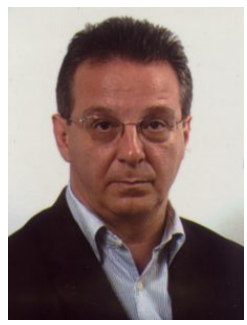

Giovanni Magenes (M'90-SM'18) was born in Genoa, Italy, in 1956. He received the M.S. degree (cum laude) in electrical engineering from the University of Pavia, Pavia, Italy, in 1981 and the Ph.D. degree in biomedical engineering from Politecnico di Milano, Milan, Italy, in 1987.

He was a Visiting Researcher with the Institut Nationale de la Santé et Réchèrche Medicale, Lyon, France, and a Visiting Professor with the Faculté des Sciences, Université de la Mediterraneé, Luminy, France. He is currently a Full Professor of biomedical signal and image processing with the Faculty of Engineering, University of Pavia, where he is also the Director of the Centre for Health Technologies (CHT). He is also with the Technological Innovation Section, European Centre for Training and Research in Earthquake Engineering, Pavia. He has been involved in various international and national research projects in the field of biomedical engineering. His research interests include soft computing methods in biomedical applications, biomedical signal and image processing, hybrid tissues, and wearable devices. 